\title{
Disección espontánea de la arteria descendente anterior en gestante con desprendimiento prematuro de placenta normoinserta. Reporte de caso
}

\section{Case report Spontaneous descending artery dissection in pregnant woman with premature placental abruption}

Leticia Duarte ${ }^{1}$, Patricia Balián², Ana Laura Cebriá1, Martín Pérez De Palleja³, Juan Pablo Bouchacourt ${ }^{4}$

\begin{abstract}
Coronary disease is infrequent among young population. Diagnosis in the obstetric population is not easy, due to the physiological changes that this population presents. Spontaneous coronary artery dissection, not a frequent medical condition, can be a cause of myocardial ischemia and be associated with and acute coronary syndrome. We present the case of a 38-year-old female patient, at 33 weeks gestation, who presented an acute coronary syndrome with ST segment elevation, caused by coronary artery dissection. This syndrome was accompanied with abruptio placentae and fetal death.
\end{abstract}

\section{RESUMEN}

La patología coronaria no es frecuente dentro de la población joven. El diagnóstico en la paciente obstétrica no es fácil dado los cambios fisiológicos que esta población presenta. La disección espontánea de una arteria coronaria es una afección muy poco frecuente que puede ser causa de isquemia miocárdica y asociarse a un síndrome coronario agudo. Se trata del caso de una paciente de sexo femenino de 38 años cursando 33 semanas de gestación, la cual presentó un síndrome coronario agudo con elevación del segmento ST, por una disección coronaria, desprendimiento prematuro de placenta normo inserta (DPPNI) y óbito fetal.
Key words:

Obstetrics,

coronary disecction, abruptio placentae

\section{Palabras clave:}

Obstetricia, disección coronaria, desprendimiento de placenta

Médico Anestesiólogo. Asistente del Departamento de Anestesiología. Hospital de Clínicas, Facultad de Medicina, UDELAR. Montevideo, Uruguay.

Médico Anestesiólogo

Médico Anestesiólogo. Asistente del Departamento de Anestesiología. Hospital de Clínicas, Facultad de Medicina, UDELAR, Montevideo, Uruguay. Fellow en Anestesia Obstétrica World Federation of Societies of Anesthesiologists.

4 Médico Anestesiólogo. Profesor Agregado del Departamento de Anestesiología. Hospital de Clínicas, Facultad de Medicina, UDELAR. Montevideo, Uruguay.

Fecha de recepción: 03 de mayo de 2018

Fecha de aceptación: 30 de junio de 2019

ORCID

https://orcid.org/0000-0002-0761-3251

Correspondencia:

Dr. Juan Pablo Bouchacourt

Email: jboucha@hc.edu.uy 


\section{Introducción}

a disección espontánea de una arteria coronaria es una condición muy poco frecuente que puede - ser causa de isquemia miocárdica y asociarse a un síndrome coronario agudo[1]. A su vez, el infarto agudo de miocardio durante el embarazo se asocia a una alta mortalidad materno-fetal[2]. La presentación del cuadro varía desde la angina inestable hasta la muerte súbita[3]. Los conocimientos de esta entidad se reducen a casos aislados y la ausencia de grandes series hace que su tratamiento y sus implicancias pronósticas no estén plenamente establecidas.

El diagnóstico oportuno requiere de una alta sospecha y enfoque multidisciplinario. No se debe demorar el estudio angiográfico y de ser posible realizar el ultrasonido intravascular[4]. Estas medidas afectan el pronóstico y manejo, dado que la cantidad de miocardio isquémico que se logre rescatar está inversamente relacionado al tiempo que permanezca estenosada la luz del vaso.

Para el equipo anestésico se trató en primera instancia de una paciente de alto riesgo cardiovascular que requeriría de tromboprofilaxis, en el contexto de una urgencia obstétrica dada por una hemorragia placentaria en curso; realizándose un manejo multidisciplinario complejo por lo intrincado de las patologías mencionadas.

\section{Caso clínico}

Mujer de 38 años multípara, con antecedentes personales de tabaquismo, enfermedad pulmonar obstructiva crónica, insuficiencia tricúspide leve, mi- grañas y depresión.

Acude al servicio de urgencia de un centro materno-infantil de segundo nivel de atención. Su motivo inicial de consulta fue por un síncope, posterior al cual sufrió una caída a nivel en el baño de su domicilio, resultando en un traumatismo supra ciliar derecho, con posterior amnesia del episodio. A su ingreso se constata embarazo de 33 semanas bien controlado y mal tolerado por diabetes gestacional en tratamiento con dieta. Se había constatado polihidramnios a la semana 27. En el servicio de urgencia comienza con disnea y dolor precordial por lo que se decide hospitalizarla catalogando el cuadro como trastorno conversivo. En ese momento estaba sin contracciones ni genitorragia y se constatan latidos fetales normales. En sala se realiza electrocardiograma (ECG) (Figura 1a).

Se solicitó interconsulta a anestesiología del centro dado que la paciente persiste con dolor torácico intenso, no irradiado, sudoración, hipotensión arterial y taquicardia. Se realiza lateralización del útero inmediatamente, aporte de volumen y oxigenoterapia.

Posterior a las medidas instauradas presentó leve mejoría aunque taquicárdica. Se solicitó al equipo obstétrico valoración fetal por ecografía constatándose óbito fetal y hematoma placentario de $92 \times 26 \mathrm{~cm}$ parcialmente organizado en sector medio. No tenía contracciones ni genitorragia y el cuello uterino estaba en posición posterior y cerrado. Paciente persistió con dolor torácico, evidenciándose cambios en el monitor ECG (Figuras 1 b y 1c), administrándose un comprimido sublingual de ácido acetil salicílico (AAS) de $200 \mathrm{mg}$.

De los resultados de laboratorio destacaba: tiempo de protrombina y tiempo de sangría normales, fibrinógeno de $606 \mathrm{mg} / \mathrm{dl}$, hemoglobina de $12,5 \mathrm{~g} /$

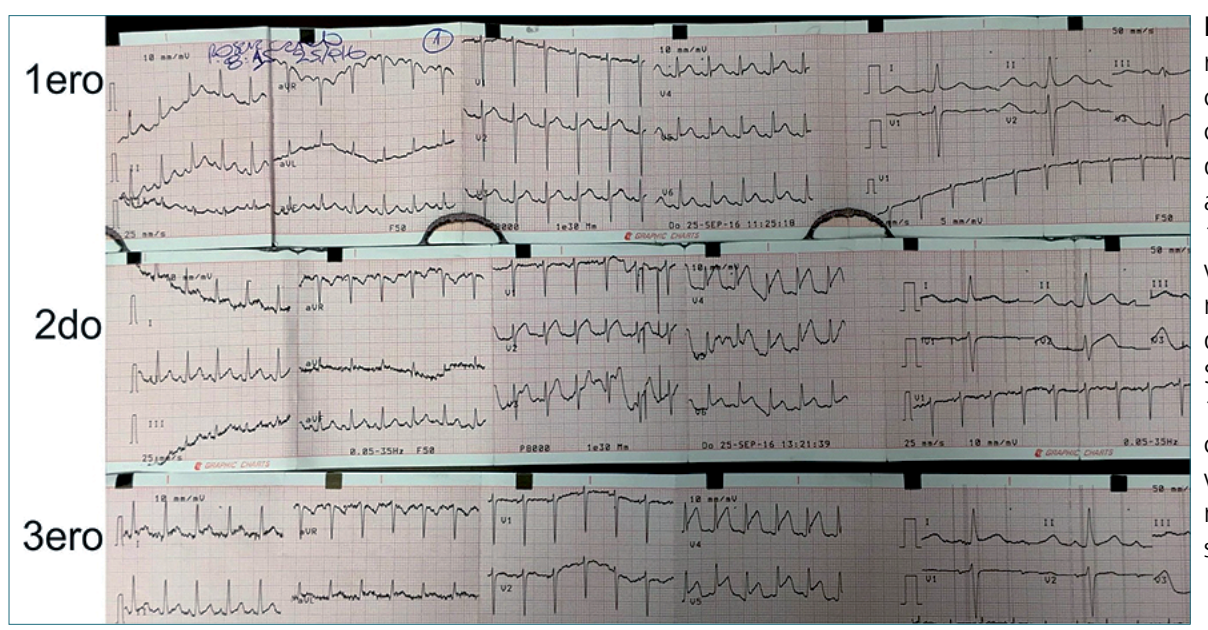

Figura 1. 1a: Se aprecia ritmo sinusal con taquicardia supra ventricular con imagen de supra desnivel del ST de V3 a V5.

1b: Taquicardia supra ventricular de 135 lat/ min, y aumento del desnivel del segmento ST V2 a V6.

1c: Mismo ritmo sinusal con taquicardia supra ventricular y mayor aumento del desnivel del segmento ST. 
dl, glóbulos blancos de $21.000 \times \mathrm{mm}^{3}$, plaquetas $339.000 \times \mathrm{mm}^{3}$, función hepática e ionograma normal. Creatin kinasa 65U/l, creatin kinasa MB 18U/l y troponinas menor a 0,01 $\mathrm{gg} / \mathrm{L}$.

Con diagnóstico de síndrome coronario agudo (SCA) con elevación del segmento ST, óbito fetal y desprendimiento prematuro de placenta normo inserta (DPPNI) estable, se coordina traslado a un centro de mayor complejidad decidiéndose en conjunto con equipo obstétrico, postergar la cesárea hasta resolución del cuadro cardiovascular o de presentar una emergencia obstétrica.

Previo al traslado, comienza con dolor abdominal y contracciones escasas, de leve intensidad, sin cambios a nivel del cuello uterino.

Se trasladó e ingresó a sala de hemodinamia donde se realizó una cineangiocoronariografía (CACG) (Figura 2).

Durante el procedimiento, la paciente presentó contracciones y genitorragia, por lo que se suspendió el mismo e ingresó al quirófano con un ECG con nivelación del segmento ST y hemodinámicamente estable. Se indujo una anestesia general para cesárea de urgencia, con monitorización estándar, además de una línea arterial radial izquierda previa a la inducción anestésica para valorar cambios hemodinámicos y tener una vía rápida para la extracción de muestras sanguíneas. La inducción anestésica fue con fentanyl, etomidato y succinilcolina para intubar la tráquea y mantención con gases halogenados. Posterior a la inducción se instaló una vía venosa central yugular derecha ecoguiada para administrar ionotrópicos y vasoconstrictores en caso necesario. La anestesia y cirugía transcurrieron sin incidentes. El sangrado fue el habitual para el procedimiento.

Luego de terminado el acto quirúrgico, volvió al centro de hemodinamia, intubada, con sedo analgesia, para tratamiento de su patología coronaria.

Al completar el estudio de CACG con ultrasonografía intracoronaria (IVUS) (Figura 3), la paciente persistió sin elevación del segmento ST y dado los hallazgos de este estudio se decidió continuar con tratamiento médico, sin necesidad de colocación de un stent. En todo momento se mantuvo hemodinámicamente estable. Permaneció en la Unidad de Cuidados Intermedios cardiológicos y se extubó la tráquea sin incidentes en la misma noche.

Se realizó ecocardiograma que informó hipoquinesia septal apical con patrón de llenado del ventrículo izquierdo normal, fracción de eyección del ventrículo izquierdo (FEVI) de 53\%, válvula tricúspide con insuficiencia leve que permitió estimar la presión arterial pulmonar (PAPS) en $22 \mathrm{mmHg}$.

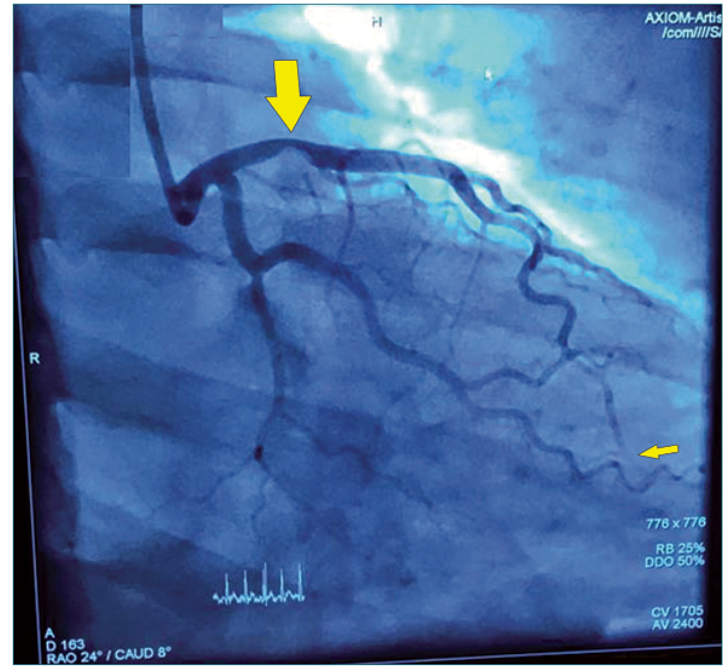

Figura 2. Coronariografía donde se aprecia flap de disección, de la cual se destaca arteria descendente anterior con buen calibre y desarrollo, presenta estenosis severa proximal de $80 \%$ (flecha grande). A nivel del tercio medio distal presenta acentuación de su flexuosidad configurando imagen en tirabuzón, el flujo es normal hasta el ápex donde se enlentece bruscamente (flecha chica). Imagen que sugiere disección coronaria espontánea con hematoma mural.

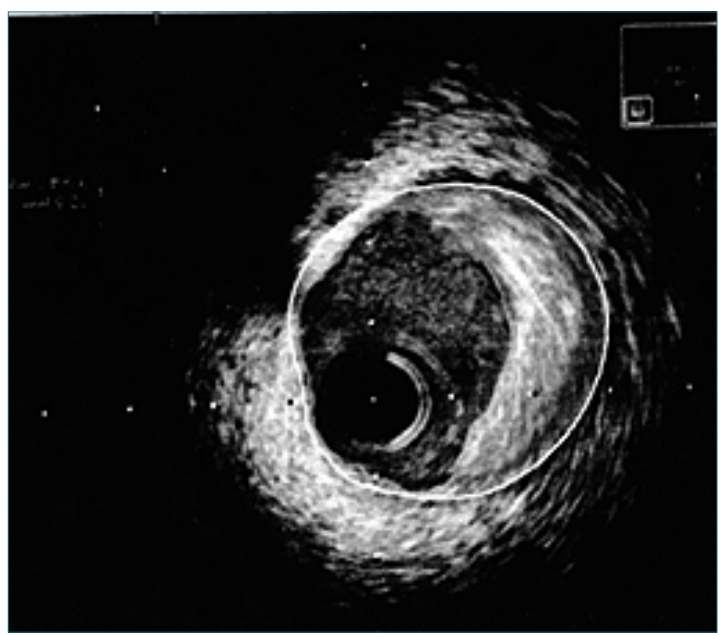

Figura 3. Ultrasonido intravascular confirmando disección de la arteria coronaria descendente anterior. Informa disección coronaria espontánea y hematoma mural, estenosis moderada de arteria descendente anterior proximal con (área luminal mínima) ALM 6,93 mm².

Se valoró la situación clínica de la paciente en forma interdisciplinaria, considerando que la colocación del stent en esta paciente que se encontraba asinto- 
mática con nivelación del segmento ST y que estaba cursando un postoperatorio inmediato en el contexto de un DPPNI no aportaba grandes beneficios, aunque sí la instauración del tratamiento médico.

Tres meses posteriores al cuadro, la paciente se encuentra en su domicilio con buena evolución gíneco-obstétrica, sin limitaciones en su vida diaria, con una buena capacidad funcional. Ha reiterado 2 consultas por síndromes coronarios agudos sin elevación del segmento ST, las cuales muestran inversión de la onda T de $\mathrm{V} 1$ a $\mathrm{V} 6$, repitiéndose CACG por los antecedentes, pero sin hallazgos ni necesidad de tratamiento intervencional. La paciente está actualmente en tratamiento con aspirina, atorvastatina y clopidogrel.

\section{Discusión}

Nos encontramos ante un caso clínico, en el cual se superponen un síndrome coronario agudo con elevación del segmento ST en evolución, al mismo tiempo que una urgencia ginecológica como lo es el DPPNI. La hemorragia al momento del ingreso se encontraba contenida aunque con el riesgo de desestabilizarse pudiendo transformarse en una emergencia. A su vez la paciente consulta en un centro sin los recursos apropiados para realizar un tratamiento integral, por lo que la necesidad inminente del traslado aumentaba aún más el riesgo materno.

Para el equipo anestesiológico que asistió a esta paciente se trató, en primera instancia de una paciente con muy alto riesgo cardiovascular, presentando una condición cardíaca activa, la cual requiere de tratamiento previo al procedimiento anestésico-quirúrgico[5], si este no es una urgencia.

Se resolvió primar la resolución de su cuadro agudo cardiovascular, dado que, por tratarse de un óbito fetal, el cuadro obstétrico no apresuraba y el desprendimiento placentario se encontraba contenido. Motivaron esta conducta que los estudios ecográficos no evidenciaban aumento del desprendimiento, los estudios de laboratorio no evidenciaban caída en los valores de hemoglobina y el centro de tercer nivel al cual se trasladaría se encontraba a diez minutos de distancia. Luego de la constatación del óbito fetal, se priorizó la resolución de la condición cardiaca activa previo al acto anestésico-quirúrgico, con el objetivo de reducir el riesgo de morbimortalidad de la paciente.

La disección coronaria es una entidad muy poco frecuente como causa de isquemia coronaria. Fue descrita por primera vez en 1931 en una paciente con muerte súbita, reportándose más de 150 casos posteriormente[6]. Se describe como la causa de 0,1\% a $4 \%$ de los síndromes coronarios agudos y como 0,4\% de las causas de muerte súbita. Las mujeres fumadoras han duplicado el riesgo de infarto de miocardio[7], como en el caso de nuestra paciente. La incidencia angiográfíca reportada varía entre 0,04 y 0,2\%[8]. La disección de las arterias coronarias genera la separación de las diferentes capas de la pared arterial, con la consecuente formación de un falso lumen. El plano de disección puede ubicarse entonces entre la íntima y la media, o entre la capa media y la adventicia. La hemorragia en el falso lumen puede comprimir la luz verdadera, resultando en una obstrucción oclusiva o no oclusiva del flujo sanguíneo. La arteria descendente anterior es el vaso que con mayor frecuencia se diseca en las mujeres, mientras que en los hombres es la coronaria derecha.

La patogenia de esta afección no está del todo establecida. Puede ser desencadenada de forma primaria (espontánea) o secundaria a traumatismos torácicos, iatrogenia (cateterismos), disección aórtica o cirugía cardíaca. En los casos relacionados con el trabajo de parto y el puerperio se han descrito infiltrados pericoronarios eosinofílicos en la autopsia, que podría relacionarse con el efecto de los estrógenos y la progesterona que estimulan la secreción eosinofílica de gránulos con sustancias líticas. La disección coronaria espontánea se ha relacionado con el embarazo debido a un exceso de progesterona que conlleva cambios bioquímicos y estructurales en la pared arterial como la pérdida normal de fibras elásticas, la fragmentación de fibras reticulares o disminución de ácidos mucopolisacáridos[9].

Otros factores que se han relacionado con la disección coronaria espontánea son la hipertensión arterial, el consumo de cocaína, el tabaquismo, historia de espasmo coronario por aumento de las fuerzas de cizallamiento en el vaso, vasculitis, síndrome de Marfán, así como otros factores hereditarios[3]. De todos estos factores de riesgo, nuestra paciente solo presentaba el antecedente de tabaquismo. Del interrogatorio dirigido a la paciente y su pareja no se desprendían elementos para sospechar consumo de cocaína ni otras drogas, por lo cual no se realizó análisis toxicológico.

El diagnóstico de infarto al miocardio en la embarazada se establece con los mismos criterios clínicos, electrocardiográficos y enzimáticos que en el resto de la población[10]. El diagnóstico específico de disección coronaria se realiza mediante coronariografía, por la presencia de una doble luz separada por un flap intimal radiotransparente. 
Dada la infrecuencia de esta patología y que la mayoría de los datos obtenidos son de necropsias[1], no podemos identificar claramente una secuencia de los acontecimientos sucedidos en esta paciente.

En conclusión, se trata de una entidad poco fre- cuente, no habiéndose encontrado reporte de casos de pacientes que presentan la asociación de disección coronaria con DPPNI y que hayan sobrevivido. El diagnóstico oportuno y el tratamiento precoz son esenciales para una buena evolución.

\section{Referencias}

1. Berenguer $A$, Mainar $V$, Bordes P, Valencia J, Arrarte V. Disección espontánea de arterias coronarias como causa infrecuente de síndromes coronarios agudos. Rev Esp Cardiol. 2003 Oct;56(10):101721. https://doi.org/10.1016/ S0300-8932(03)77001-8 PMID:14563298

2. Martínez-Quintana E, Rodríguez-González F. Embarazo y disección arterial coronaria. Clin Investig Arterioscler. 2015 Jul-Aug;27(4):215-9. PMID:25795261

3. Romero-Rodriguez N, Fernandez-Quero M, Villa Gil-Ortega M, Urbano del Moral JA, Ballesteros Prada S, Díaz de la Llera L. Disección coronaria espontanea y sus implicaciones pronósticas a largo plazo en una cohorte de 19 casos. Rev Esp Cardiol. 2010;63(9):108891. https://doi.org/10.1016/ S0300-8932(10)70231-1 PMID:20804705

4. Magarkar V, Lathi P. A case of spontaneous coronary artery dissection in early pregnancy managed by $\mathrm{PCl}$. Indian Heart J. 2016 Sep;68 Suppl 2:S25-

7. https://doi.org/10.1016/j. ihj.2016.04.021 PMID:27751306

5. Kristensen SD, Knuuti J, Saraste A, Anker S, Bøtker HE, Hert SD, et al.; Authors/Task Force Members. 2014 ESC/ESA Guidelines on non-cardiac surgery: cardiovascular assessment and management: The Joint Task Force on non-cardiac surgery: cardiovascular assessment and management of the European Society of Cardiology (ESC) and the European Society of Anaesthesiology (ESA). Eur Heart J. 2014 Sep;35(35):2383-431. https://doi.org/10.1093/eurheartj/ehu282 PMID:25086026

6. García Acuña JM, González Juanatey JR, López Lago A, Amaro Cendón A, Virgos Lamela A, Gil de la Peña M. Disección coronaria espontánea: aspectos diagnósticos y terapéutica. Med Intensiva. 2003;27(3):188-90. https://doi.org/10.1016/\$02105691(03)79891-X.
7. Hind N. Moussa, Malahat Movahedian, Mateo G. Leon, Baha M. Sibai. Acute Myocardial Infarction Due to Coronary Artery Dissection in the Postpartum Period. AJP Rep. 2015;5:e93-6.

8. Fajardo AL, Díaz P. FACC, Un desafío clínico: la disección coronaria. Rev Urug Cardiol. 2014;29:345-8.

9. Salari A, Ghilipur M. Maedeh Rezaeidanesh., Anoosh Barzigar., Sharam Rahmani., Mohadeseh Pursadeghi., Hannan Ebrahimi. A 36-Year-Old Woman with Coronary Artery Dissection Two Weeks after Abortion. J Tehran Univ Heart Cent. 2015;11(2):98-101.

10. Lipman $S$, Cohen S, Einav S, Jeejeebhoy F, Mhyre JM, Morrison LJ, et al.; Society for Obstetric Anesthesia and Perinatology. The Society for Obstetric Anesthesia and Perinatology consensus statement on the management of cardiac arrest in pregnancy. Anesth Analg. 2014 May;118(5):100316. https://doi.org/10.1213/ ANE.0000000000000171 PMID:24781570 